\title{
Exact solution of bending problem of clamped orthotropic rectangular thin plates
}

\author{
Chen An $\cdot$ Jijun Gu $\cdot$ Jian Su
}

Received: 9 January 2012 / Accepted: 30 October 2012 / Published online: 26 March 2015

(C) The Brazilian Society of Mechanical Sciences and Engineering 2015

\begin{abstract}
The generalized integral transform technique (GITT) is employed to obtain an exact solution for the bending problem of fully clamped orthotropic rectangular thin plates. The use of the GITT approach in the analysis of the transverse deflection equation leads to a coupled system of fourth order differential equations (ODEs) in the dimensionless longitudinal spatial variable. The resulting transformed ODE system is then numerically solved by making use of the subroutine DBVPFD from IMSL Library. Numerical results with automatic global accuracy control are obtained for different values of aspect ratio. Critical comparisons with previously reported numerical results are performed with excellent agreement. A set of reference results for clamped orthotropic rectangular plates is also provided for future covalidation purposes, which are verified by those obtained from the general-purpose finite element software package ABAQUS.
\end{abstract}

Keywords Orthotropic rectangular thin plates · Bending · Integral transform $\cdot$ Exact solution

Technical Editor: Lavinia Borges.

C. An $(\bowtie) \cdot$ J. Gu

Offshore Oil/Gas Research Center, China University

of Petroleum-Beijing, Beijing 102249, China

e-mail: anchen@cup.edu.cn

J. Gu

e-mail: carson817@gmail.com

J. Su

Nuclear Engineering Program, COPPE, Universidade Federal do Rio de Janeiro, CP 68509, Rio de Janeiro 21941-972, Brazil e-mail: sujian@nuclear.ufrj.br

\section{Introduction}

The bending problem of orthotropic rectangular plates with clamped edges has been studied over the past century, due to its relevance in engineering [1]. Many different methods have been studied to solve the plate bending problems. Recently, Li et al. [2] proposed a double finite sine integral transform method to obtain exact bending solutions of such plates under arbitrary loadings without any trial functions. Zhong and $\mathrm{Li}$ [3] developed a new symplectic method for analysis of a rectangular thin plate with all edges clamped, and also obtained the exact solutions. In addition, using Galerkin method, Mbakogu and Pavlovic [4] obtained an approximate solution by means of Mathematica [5]. Dalaei and Kerr [6] and Aghdam and Falahatgar [7] utilized the extended Kantorovich method to generate a closed-form approximate solution for the deflections of a clamped orthotropic plate subjected to a uniformly distributed load for various aspect ratios. Meleshko [1] presented a detailed review of the superposition methods and provided a simplification of the numerical algorithm. Donning and Liu [8] developed the meshless method to analyze a clamped thick circular plate with a uniform load using Mindlin-Reissner theory. Papargyri-Beskou et al. [9] assessed analytically the static response of a clamped circular gradient elastic plate based on the variational formulation with the aid of the principle of virtual work. Besides, for the purpose of analyzing moderately thick plates with the transverse shear effects, a spline finite element method was developed by Shen and He [10]. Adopting the method of modified two-variable and the method of mixing perturbation, the problems of the nonlinear unsymmetrical bending for orthotropic rectangular thin plate with variable thickness was studied by Huang [11]. Furthermore, Civalek [12] proposed a 
discrete singular convolution approach to give a numerical solution of three-dimensional analysis of thick rectangular plates.

In this work, we applied the generalized integral transform technique (GITT) to derive the exact bending solution of fully clamped orthotropic rectangular thin plates. Unlike the double finite sine integral transform method proposed by Li et al. [2], GITT is mathematically more general, which has been successfully developed in heat and fluid flow applications [13-15]. The most interesting feature of this technique is the automatic and straightforward global error control procedure, which makes it particularly suitable for benchmarking purposes, and the only mild increase in overall computational effort with increasing number of independent variables. The GITT has been applied in analyzing the dynamic response of axially moving strings [16], axially moving beams [17], damaged Euler-Bernoulli beams [18], cantilever beams with an eccentric tip mass [19], axially moving Timoshenko beams [20], axially moving orthotropic plates [21] and fluid-conveying pipes [22], the wind-induced vibration of overhead conductors [24], and predicting the vortex-induced vibration of long flexible cylinders [23], which demonstrated high accuracy, excellent convergence behavior and good long-time numerical stability of the approach. However, to the authors' best knowledge, no previous study has performed the bending analysis of fully clamped orthotropic rectangular plates based on GITT approach. The paper is organized as follows. In the next section, the mathematical formulation of the problem of a uniformly loaded orthotropic rectangular plate with clamped edges is presented. In the following section, the exact analytical solution is obtained by carrying out integral transform. Numerical results with automatic global accuracy control are then presented for different values of aspect ratio. A comparison against the previous work [25, 26] is also performed to assess the accuracy and convergence of the present approach. Finally, a fully clamped orthotropic plate is examined, aiming at bringing a set of benchmark results for future comparison. The results demonstrate excellent accuracy when compared with those obtained using commercial finite element code ABAQUS/Standard 6.9-1 [27].

\section{Mathematical formulation}

Consider an orthotropic rectangular thin plate with constant thickness and all four edges clamped, as illustrated in Fig. 1. The principle axes of orthotropy coincide with the $x$ and $y$ direction. The bending problem is governed by the following equation $[25,28]$ :

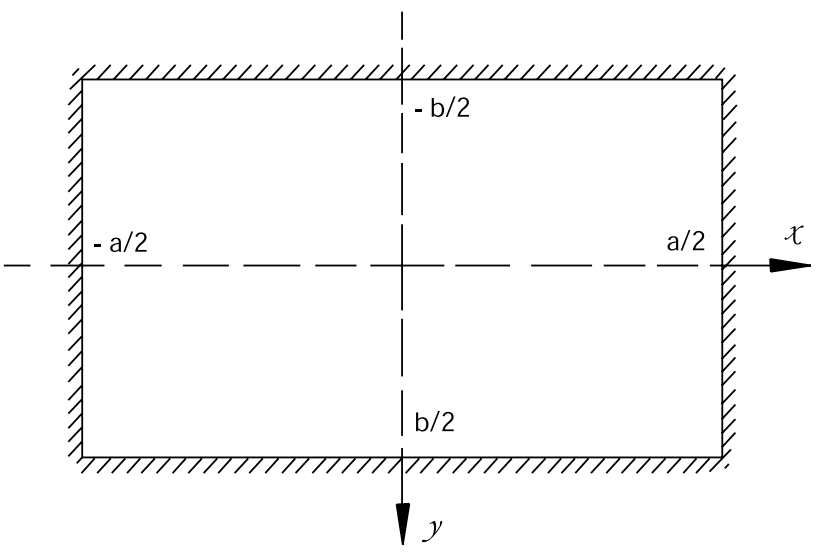

Fig. 1 Clamped orthotropic rectangular plate under consideration

$D_{x} \frac{\partial^{4} w}{\partial x^{4}}+2 H \frac{\partial^{4} w}{\partial x^{2} \partial y^{2}}+D_{y} \frac{\partial^{4} w}{\partial y^{4}}=q$,

where $w$ and $q$ are the transverse deflection of the plate and the applied transverse loading, respectively. $D_{x}$ and $D_{y}$ represent the flexural rigidities about the $y$ and $x$ axes, respectively. $H$, the effective torsional rigidity, is given by $H=D_{1}+2 D_{x y}$, where $D_{x y}$ denotes the torsional rigidity and $D_{1}$ is defined in terms of the reduced Possion's ratios $v_{1}$ and $v_{2}$, as $D_{1}=v_{2} D_{x}=v_{1} D_{y}$.

Furthermore, the bending moments $M_{x}$ and $M_{y}$, the torsional moment $M_{x y}$, the transverse shear forces $Q_{x}$ and $Q_{y}$ can be determined by the following relations:

$M_{x}=-\left(D_{x} \frac{\partial^{2} w}{\partial x^{2}}+D_{1} \frac{\partial^{2} w}{\partial y^{2}}\right)$

$M_{y}=-\left(D_{1} \frac{\partial^{2} w}{\partial x^{2}}+D_{y} \frac{\partial^{2} w}{\partial y^{2}}\right)$

$M_{x y}=-2 D_{x y} \frac{\partial^{2} w}{\partial x \partial y}$,

$Q_{x}=-\frac{\partial}{\partial x}\left(D_{x} \frac{\partial^{2} w}{\partial x^{2}}+H \frac{\partial^{2} w}{\partial y^{2}}\right)$,

$Q_{y}=-\frac{\partial}{\partial y}\left(H \frac{\partial^{2} w}{\partial x^{2}}+D_{y} \frac{\partial^{2} w}{\partial y^{2}}\right)$.

In the specific case of isotropy, we have $v_{1}=v_{2}=v$, $D_{x}=D_{y}=H=D, \quad D_{1}=v D \quad$ and $\quad D_{x y}=(1-v) D / 2$, where the symbols $D$ and $v$ represent the flexural rigidity and Poisson's ratio. With these relations, the foregoing equations can be considerably simplified. 
The boundary conditions for such problem are given by

$$
w=\frac{\partial w}{\partial x}=0, \quad \text { at } \quad x= \pm \frac{a}{2} \quad \text { and } \quad w=\frac{\partial w}{\partial y}=0, \quad \text { at } \quad y= \pm \frac{b}{2} .
$$

Equation (1) and boundary conditions (3) complete the mathematical formulation of the problem. By introducing the following dimensionless parameters

$$
\begin{aligned}
& \xi=\frac{x}{a / 2}, \quad \eta=\frac{y}{b / 2}, \quad c=\frac{b}{a}, \\
& \alpha=\frac{H}{D_{x}}, \quad \beta=\frac{D_{y}}{D_{x}}, \quad \tilde{w}=\frac{w}{a^{4} q / D_{x}},
\end{aligned}
$$

the mathematical formulation in dimensionless form can be written as follows

$\frac{\partial^{4} \tilde{w}}{\partial \xi^{4}}+\frac{2 \alpha}{c^{2}} \frac{\partial^{4} \tilde{w}}{\partial \xi^{2} \partial \eta^{2}}+\frac{\beta}{c^{4}} \frac{\partial^{4} \tilde{w}}{\partial \eta^{4}}=\frac{1}{16}$.

The boundary conditions in dimensionless form are written as

$\tilde{w}(-1, \eta)=0, \quad \frac{\partial \tilde{w}(-1, \eta)}{\partial \xi}=0, \quad \tilde{w}(1, \eta)=0, \quad \frac{\partial \tilde{w}(1, \eta)}{\partial \xi}=0$,

$\tilde{w}(\xi,-1)=0, \quad \frac{\partial \tilde{w}(\xi,-1)}{\partial \eta}=0, \quad \tilde{w}(\xi, 1)=0, \quad \frac{\partial \tilde{w}(\xi, 1)}{\partial \eta}=0$.

\section{Integral transform solution}

Following the ideas in the generalized integral transform technique [29], the auxiliary eigenvalue problems needed for the integral transformation process are chosen from homogeneous versions of the original problems. For the problem (5), the eigenvalue problem is given as

$\frac{\mathrm{d}^{4} Y_{i}(\eta)}{\mathrm{d} \eta^{4}}=\mu_{i}^{4} Y_{i}(\eta), \quad-1<\eta<1$,

with the following boundary conditions

$Y_{i}(-1)=0, \quad \frac{\mathrm{d} Y_{i}(-1)}{\mathrm{d} \eta}=0, \quad Y_{i}(1)=0, \quad \frac{\mathrm{d} Y_{i}(1)}{\mathrm{d} \eta}=0$,

where $Y_{i}(\eta)$ and $\mu_{i}$ are, respectively, the eigenfunctions and eigenvalues of problem (6), which satisfy the following orthogonality property

$\int_{-1}^{1} Y_{i}(\eta) Y_{j}(\eta) \mathrm{d} \eta=\delta_{i j} N_{i}$,

Problem (6) is readily solved analytically to yield

$Y_{i}(\eta)= \begin{cases}\frac{\cos \left(\mu_{i} \eta\right)}{\cos \left(\mu_{i}\right)}-\frac{\cosh \left(\mu_{i} \eta\right)}{\cosh \left(\mu_{i}\right)}, & \text { for i odd, } \\ \frac{\sin \left(\mu_{i} \eta\right)}{\sin \left(\mu_{i}\right)}-\frac{\sinh \left(\mu_{i} \eta\right)}{\sinh \left(\mu_{i}\right)}, & \text { for i even, }\end{cases}$ and the transcendental equation for the eigenvalues becomes

$\tanh \left(\mu_{i}\right)= \begin{cases}-\tan \left(\mu_{i}\right), & \text { for i odd, } \\ \tan \left(\mu_{i}\right), & \text { for i even, }\end{cases}$

The norm, or normalization integral, is written as

$N_{i}=\int_{-1}^{1} Y_{i}^{2}(\eta) \mathrm{d} \eta=2, \quad i=1,2,3, \ldots$

The eigenvalue problem (6) allows definition of the following integral transform pair

$\bar{w}_{i}(\xi)=\int_{-1}^{1} \tilde{Y}_{i}(\eta) \tilde{w}(\xi, \eta) \mathrm{d} \eta, \quad$ transform,

$\tilde{w}(\xi, \eta)=\sum_{i=1}^{\infty} \tilde{Y}_{i}(\eta) \bar{w}_{i}(\xi), \quad$ inverse

where $\tilde{Y}_{i}(\eta)$ is the normalized eigenfunction

$\tilde{Y}_{i}(\eta)=\frac{Y_{i}(\eta)}{N_{i}^{1 / 2}}=\frac{Y_{i}(\eta)}{\sqrt{2}}$.

Now, to perform the integral transform process, the dimensionless equation (5a) is multiplied by the operator $\int_{-1}^{1} \tilde{Y}_{i}(\eta) \mathrm{d} \eta$ and the inverse formula (11b) is applied. After some mathematical manipulations, the following set of ordinary differential equations can be obtained:

$\frac{\mathrm{d}^{4} \bar{w}_{i}}{\mathrm{~d} \xi^{4}}+\frac{2 \alpha}{c^{2}} \sum_{j=1}^{\infty} D_{i j} \frac{\mathrm{d}^{2} \bar{w}_{j}}{\mathrm{~d} \xi^{2}}+\frac{\beta}{c^{4}} \mu_{i}^{4} \bar{w}_{i}=\frac{1}{16} Q_{i}, \quad i=1,2,3, \ldots$,

where the coefficients are analytically determined from the following integrals

$D_{i j}=\int_{-1}^{1} \tilde{Y}_{i} \frac{\mathrm{d}^{2} \tilde{Y}_{j}}{\mathrm{~d} \eta^{2}} \mathrm{~d} \eta, \quad Q_{i}=\int_{-1}^{1} \tilde{Y}_{i} \mathrm{~d} \eta$.

In a similar manner, the boundary conditions are also integral transformed in the $\eta$ direction to yield

$\bar{w}_{i}(-1)=0, \quad \frac{\mathrm{d} \bar{w}_{i}(-1)}{\mathrm{d} \xi}=0, \quad \bar{w}_{i}(1)=0, \quad \frac{\mathrm{d} \bar{w}_{i}(1)}{\mathrm{d} \xi}=0$.

$(14 a-d)$

The integral transformation then eliminates the coordinate $\eta$ and Eqs. (13) and (14) offer an ODE system in the $\xi$ direction for the transformed potentials $\bar{w}_{i}(\xi)$, subjected to the corresponding integral transformed boundary conditions.

For computational purposes, the coefficients $(13 b, c)$ are computed through the software of symbolic manipulation Mathematica [5]. In order to solve the ordinary differential system, infinite summations should be truncated to a 
Table 1 Convergence of transverse deflections $\left.w\right|_{x=0, y=0}\left(q_{0} a^{4} / D\right)$, bending moments $\left.M_{x}\right|_{x=a / 2, y=0}\left(q_{0} a^{2}\right)$ and transverse shear forces $\left.Q_{x}\right|_{x=a / 2, y=0}\left(q_{0} a\right)$ for an isotropic plate under uniform load with dif- ferent truncation orders $N$, and comparison with benchmark series solution $[25,26]$

\begin{tabular}{|c|c|c|c|c|c|c|c|}
\hline$c$ & $N=4$ & $N=8$ & $N=16$ & $N=32$ & $N=64$ & $N=128$ & \\
\hline$w$ & & & & & & & Ref. [25] \\
\hline 1.0 & 0.0012608129 & 0.0012667909 & 0.0012655719 & 0.0012655844 & 0.0012655976 & 0.0012654348 & 0.00126 \\
\hline 1.2 & 0.0017145927 & 0.0017239501 & 0.0017272244 & 0.0017272646 & 0.0017272654 & 0.0017272653 & 0.00172 \\
\hline 1.4 & 0.0020490712 & 0.0020693270 & 0.0020704414 & 0.0020705126 & 0.0020682660 & 0.0020681748 & 0.00207 \\
\hline 1.6 & 0.0022692459 & 0.0023000098 & 0.0022998904 & 0.0023002145 & 0.0023002184 & 0.0023002173 & 0.00230 \\
\hline 1.8 & 0.0024011140 & 0.0024446135 & 0.0024460384 & 0.0024463674 & 0.0024463616 & 0.0024463604 & 0.00245 \\
\hline 2.0 & 0.0024711022 & 0.0025294342 & 0.0025343818 & 0.0025332840 & 0.0025331015 & 0.0025331099 & 0.00254 \\
\hline$\infty$ & 0.0021043499 & 0.0023319225 & 0.0024625838 & 0.0025320938 & 0.0025678256 & 0.0025859228 & 0.00260 \\
\hline$M_{x}$ & & & & & & & Ref. [25] \\
\hline 1.0 & -0.051057804 & -0.051421266 & -0.051369518 & -0.051340580 & -0.051335275 & -0.051334161 & -0.0513 \\
\hline 1.2 & -0.063298516 & -0.063984588 & -0.063950464 & -0.063913926 & -0.063906506 & -0.063905440 & -0.0639 \\
\hline 1.4 & -0.071514159 & -0.072656088 & -0.072659284 & -0.072617583 & -0.072594114 & -0.072592147 & -0.0726 \\
\hline 1.6 & -0.076355241 & -0.078041501 & -0.078091688 & -0.078050099 & -0.078038074 & -0.078036375 & -0.0780 \\
\hline 1.8 & -0.078817144 & -0.081109077 & -0.081245355 & -0.081204608 & -0.081190348 & -0.081188165 & -0.0812 \\
\hline 2.0 & -0.079744078 & -0.082680248 & -0.082937367 & -0.082888953 & -0.082870565 & -0.082867987 & -0.0829 \\
\hline$\infty$ & -0.067339197 & -0.074621521 & -0.078802682 & -0.081027001 & -0.082170419 & -0.082749531 & -0.0833 \\
\hline$Q_{x}$ & & & & & & & Ref. [26] \\
\hline 1.00 & -0.4427811 & -0.4490769 & -0.4451593 & -0.4422929 & -0.4413742 & -0.4412414 & -0.44 \\
\hline 1.25 & -0.4880950 & -0.5009497 & -0.4980304 & -0.4949119 & -0.4938095 & -0.4935784 & -0.49 \\
\hline 1.50 & -0.4993776 & -0.5199859 & -0.5190470 & -0.5159256 & -0.5145974 & -0.5143045 & -0.52 \\
\hline 1.75 & -0.4934982 & -0.5217735 & -0.5232034 & -0.5203658 & -0.5189065 & -0.5185322 & -0.52 \\
\hline 2.00 & -0.4811883 & -0.5164700 & -0.5205043 & -0.5180333 & -0.5164853 & -0.5160354 & -0.52 \\
\hline$\infty$ & -0.4040352 & -0.4477292 & -0.4728161 & -0.4861621 & -0.4930226 & -0.4964970 & -0.50 \\
\hline
\end{tabular}

$(v=0.3)$

sufficiently large order for computational purposes. This truncated system is then solved by a computer program developed in Fortran 90, based on the use of the subroutine DBVPFD from IMSL Library [30]. This subroutine offers an interesting combination of accuracy control, and for this problem the error $10^{-8}$ is selected.

Once $\bar{w}_{i}(\xi)$ have been numerically evaluated, the analytical inversion formula (11b) recovers the dimensionless function $\tilde{w}(\xi, \eta)$. Then use the relations (4f) to obtain the desired deflection function $w(x, y)$, straightforwardly. The bending moments and the transverse shear forces can be obtained using the expressions (2).

To clarify the so-called "exact" solution, it is necessary to make final remarks about the nature of the proposed approach. Since each term of $\tilde{Y}_{i}(\eta)$ and $\bar{w}_{i}(\xi)$ satisfies boundary conditions (6b-e) and (14a-d), respectively, besides, there is no approximation involved in the analytical derivation of GITT approach, the problem solution represented by the sum of a rapidly converging infinite series (11b) simultaneously satisfies the governing differential equation (5a) and the boundary conditions ( $5 b-e)$ and ( $5 f-$ i). In real calculations, the derived series solution needs to be truncated somewhere for computational evaluation. In other words, the problem can only be dealt with through finite series instead of the infinite range in the analytical formulation. Therefore, the "exact solution" can be characterized as a truncated series solution with the desired precision, which can be achieved by controlling every numerical step with prescribed accuracy [13-15].

\section{Results and discussion}

We now present numerical results for a fully clamped isotropic rectangular thin plate with different aspect ratio $c$. In order to allow comparisons to results available in the literature [25, 26], we consider two types of load distribution: (1) a uniform load of intensity $q_{0}$ and (2) linearly varying hydrostatic pressure with the intensity $q_{1}(x / a+1 / 2)$, and the solution of the systems (13a) is obtained with $N \leq 128$ to observe the convergence behavior.

First, the numerical code is validated by making an analysis of the convergence behavior of the transverse deflection $w$ at the center point $(0,0)$ for an isotropic plate under 
Table 2 Convergence of transverse deflections $\left.w\right|_{x=0, y=0}\left(q_{1} a^{4} / D\right)$ and bending moments $\left.M_{x}\right|_{x=a / 2, y=0}\left(q_{1} a^{2}\right)$ for an isotropic plate under linearly varying hydrostatic pressure with different truncation orders $N$, and comparison with benchmark series solution [25]

\begin{tabular}{|c|c|c|c|c|c|c|c|}
\hline$c$ & $N=4$ & $N=8$ & $N=16$ & $N=32$ & $N=64$ & $N=128$ & Ref. [25] \\
\hline \multicolumn{8}{|l|}{$w$} \\
\hline 0.5 & 0.000079996891 & 0.000080089194 & 0.000080091879 & 0.000080091668 & 0.000080089580 & 0.000080086756 & 0.000080 \\
\hline $2 / 3$ & 0.00021655210 & 0.00021703285 & 0.00021706206 & 0.00021706329 & 0.00021706335 & 0.00021706292 & 0.000217 \\
\hline 1.0 & 0.00063040643 & 0.00063339545 & 0.00063357473 & 0.00063361990 & 0.00063362031 & 0.00063362031 & 0.00063 \\
\hline 1.5 & 0.0010859857 & 0.0010985999 & 0.0010994383 & 0.0010994847 & 0.0010985625 & 0.0010983938 & 0.00110 \\
\hline$\infty$ & 0.0010521750 & 0.0011659613 & 0.0012312919 & 0.0012660469 & 0.0012839128 & 0.0012929614 & 0.00130 \\
\hline \multicolumn{8}{|l|}{$M_{x}$} \\
\hline 0.5 & -0.011395857 & -0.011475792 & -0.011465784 & -0.011459917 & -0.011458791 & -0.011458686 & -0.0115 \\
\hline $2 / 3$ & -0.018574493 & -0.018732069 & -0.018720109 & -0.018710753 & -0.018708861 & -0.018708650 & -0.0187 \\
\hline 1.0 & -0.033009652 & -0.033455905 & -0.033460096 & -0.033444501 & -0.033440758 & -0.033440258 & -0.0334 \\
\hline 1.5 & -0.044831950 & -0.046050083 & -0.046163298 & -0.046148185 & -0.046136286 & -0.046134945 & -0.0462 \\
\hline$\infty$ & -0.040399440 & -0.044768393 & -0.047276840 & -0.048611298 & -0.049297273 & -0.049644697 & -0.0500 \\
\hline
\end{tabular}

$(v=0.3)$

Table 3 Deflections $\left.w\right|_{x=0, y=0}\left(q_{0} a^{4} / D_{x}\right)$, bending moments $\left.M_{x}\right|_{x=a / 2, y=0}\left(q_{0} a^{2}\right)$ and transverse shear forces $\left.Q_{x}\right|_{x=a / 2, y=0}\left(q_{0} a\right)$ for an orthotropic plate $\left(D_{y}=4 D_{x}, D_{x y}=0.85 D_{x}, v_{1}=0.075, \nu_{2}=0.3\right)$ under uniform load with truncation order $N=200$, and comparison with results obtained by ABAQUS/Standard 6.9-1 [27]

\begin{tabular}{lllllll}
\hline$c$ & $w(\mathrm{GITT})$ & $w$ (ABAQUS) & $M_{x}(\mathrm{GITT})$ & $M_{x}(\mathrm{ABAQUS})$ & $Q_{x}(\mathrm{GITT})$ & $Q_{x}(\mathrm{ABAQUS})$ \\
\hline 1.0 & 0.00052239018 & 0.000522012 & -0.028415356 & -0.0276140 & -0.3287415 & -0.324620 \\
1.2 & 0.00087223947 & 0.000871311 & -0.039689187 & -0.0387367 & -0.3891484 & -0.385162 \\
1.4 & 0.0012398233 & 0.00123981 & -0.050606117 & -0.0495232 & -0.4382915 & -0.434386 \\
1.6 & 0.0015792826 & 0.00157916 & -0.060024615 & -0.0588520 & -0.4736315 & -0.469958 \\
1.8 & 0.0018662907 & 0.00186381 & -0.067505836 & -0.0662648 & -0.4965115 & -0.493058 \\
2.0 & 0.0020880701 & 0.00208797 & -0.073074900 & -0.0718104 & -0.5097061 & -0.506490 \\
$\infty$ & 0.0025924728 & $(0.00260)^{\mathrm{a}}$ & -0.082959130 & $(-0.0833)^{\mathrm{a}}$ & -0.4977548 & $(-0.50)^{\mathrm{a}}$ \\
\hline
\end{tabular}

a The results in parentheses are from an isotropic plate, when $c$ approaches infinity [25]

uniform load with different truncation orders $N$, as shown in Table 1 . We can observe that convergence is essentially achieved with a reasonably low truncation orders $(N \leq 8)$. For a full convergence to four or six significant digits of the transverse deflection, higher orders are required $(N \leq 32$ or $N \leq 64)$. Such results provide excellent agreement when compared to the series solutions presented by Timoshenko and Woinowsky-Krieger [25]. As can be observed, convergence rate for the case of infinite aspect ratio $(c=\infty)$ is slow with the greatest value difference being $0.54 \%$ at $N=128$. Table 1 also brings the convergence behavior of the bending moments $M_{x}$ and the transverse shear forces $Q_{x}$ at the edge point $(a / 2,0)$, as well as a comparison with those results presented by Timoshenko and WoinowskyKrieger [25] and Wojtaszak [26], respectively. As would be expected, convergence rates of the bending moments are slower than ones of the transverse deflection, and faster comparing to the transverse shear forces field. This is due to the fact that the bending moments and the transverse shear forces are proportional to the second and third derivatives of the deflection function, respectively. Again, the agreement with references $[25,26]$ is essentially perfect.

Next, we perform a similar analysis about the transverse deflections and bending moments for an isotropic plate under linearly varying hydrostatic pressure $q_{1}(x / a+1 / 2)$ , as presented in Table 2. The same behavior is obtained as the previous cases. The excellent convergence rate of the transverse deflections can be noticed that an accuracy of four significant digits can be achieved with truncation orders as low as $N \leq 16$ ( $c=0.5$ and 1.5). It can be seen that, for $c=1.0$, convergence to eight significant digits of the transverse deflection is obtained with $N \leq 64$.

For future comparison, a fully clamped orthotropic plate with $D_{y}=4 D_{x}, D_{x y}=0.85 D_{x}, v_{1}=0.075$ and $v_{2}=0.3$, the same one proposed by $\mathrm{Li}$ et al. [2], is examined. Two sets of results are produced for the plate, also considering two types of load distribution: (1) a uniform load of intensity $q_{0}$ and (2) linearly varying hydrostatic pressure with 
Table 4 Deflections $\left.w\right|_{x=0, y=0}\left(q_{1} a^{4} / D_{x}\right)$, bending moments $\left.M_{x}\right|_{x=a / 2, y=0}\left(q_{1} a^{2}\right)$ and transverse shear forces $\left.Q_{x}\right|_{x=a / 2, y=0}\left(q_{1} a\right)$ for an orthotropic plate $\left(D_{y}=4 D_{x}, D_{x y}=0.85 D_{x}, v_{1}=0.075, v_{2}=0.3\right)$ under linearly varying hydrostatic pressure with truncation order $N=200$, and comparison with results obtained by ABAQUS/Standard 6.9-1 [27]

\begin{tabular}{llllllr}
\hline$c$ & $w(\mathrm{GITT})$ & $w$ (ABAQUS) & $M_{x}(\mathrm{GITT})$ & $M_{x}(\mathrm{ABAQUS})$ & $Q_{x}(\mathrm{GITT})$ & $Q_{x}(\mathrm{ABAQUS})$ \\
\hline 1.0 & 0.00026120731 & 0.000261005 & -0.020572972 & -0.0199352 & -0.2597291 & -0.256136 \\
1.2 & 0.00043622658 & 0.000435656 & -0.027072335 & -0.0263490 & -0.2937927 & -0.290342 \\
1.4 & 0.00062084210 & 0.000619905 & -0.033049017 & -0.0322579 & -0.3198004 & -0.316530 \\
1.6 & 0.00079073507 & 0.000789579 & -0.038054589 & -0.0372152 & -0.3378707 & -0.334746 \\
1.8 & 0.00093314535 & 0.000931908 & -0.041949768 & -0.0410800 & -0.3492602 & -0.346248 \\
2.0 & 0.0010441198 & 0.001043983 & -0.044816092 & -0.0439340 & -0.3556402 & -0.352760 \\
$\infty$ & 0.0012962364 & $(0.00130)^{\mathrm{a}}$ & -0.049770471 & $(-0.0500)^{\mathrm{a}}$ & -0.3484182 &
\end{tabular}

a The results in parentheses are from an isotropic plate, when $c$ approaches infinity [25]

the intensity $q_{1}(x / a+1 / 2)$, as shown in Tables 3 and 4 , respectively. In the present analysis, we use relatively high truncation orders $(N=200)$ for a sufficient accuracy.

To demonstrate the validity and the accuracy of this study, three-dimensional linear-elastic finite element analyses are also performed using the commercial software package ABAQUS/Standard 6.9-1 [27]. To ensure the applicability of the classical thin plate theory, thicknessto-span ratio of $1 / 100$ is adopted (note that "span" refers to short edge length of the rectangular plates). During the analysis, the plates are discretized using sufficiently refined S4R elements, viz., 200 elements in the $x$ direction and $200 \times c$ elements in the $y$ direction. Tables 3 and 4 show the finite element solutions of the dimensionless deflections, bending moments and transverse shear forces for the examined orthotropic plates. It can be seen that the results of the GITT approach are in excellent agreement with the results obtained by ABAQUS. As expected, the discrepancies between ABAQUS results and GITT solutions of bending moments and transverse shear forces are generally higher than those of deflections due to the higher-order derivatives involved in the expressions (2a) and (2d).

It is interesting to compare the calculated values corresponding to the limiting case $(c=\infty)$ to such results obtained from an isotropic plate [25], and the agreement between the dimensionless results can be explicitly observed. Clearly, it follows that as the aspect ratio $c$ approaches infinity, one-way beam bending (along the short edge) predominates [4].

\section{Conclusions}

The generalized integral transform technique (GITT) has proved in this paper to be a good approach for the analysis of bending problem of a fully clamped orthotropic rectangular thin plate, providing an exact numerical-analytical solution for the transverse deflections, bending moments and transverse shear forces. The numerical results obtained are in excellent agreement with the ones reported in the literature $[25,26]$ and obtained by commercial program ABAQUS. This investigation verifies that the proposed approach can be either employed for benchmarking purposes, yielding sets of reference results with controlled accuracy or, alternatively, as an engineering simulation tool with lower truncation orders and exceptional computational performances. It is important to notice that the methodology developed is applicable to any boundary conditions, and may also to the analysis of bending problems of circular cylindrical shells and multilayer composite plates.

Acknowledgments The authors acknowledge gratefully the financial support provided by CNPq, CAPES and FAPERJ of Brazil for their research work. The work was also supported by Science Foundation of China University of Petroleum-Beijing (No. 2462013YJRC003). Chen An and Jijun Gu also would like to acknowledge the financial support provided by the China Scholarship Council. Special thanks to Guang-Ming Fu of Ocean Engineering Program at COPPE/UFRJ for fruitful discussion of the results obtained by ABAQUS.

\section{References}

1. Meleshko VV (1997) Bending of an elastic rectangular clamped plate: Exact versus 'engineering' solutions. J Elast 48(1):1-50

2. Li R, Zhong Y, Tian B, Liu YM (2009) On the finite integral transform method for exact bending solutions of fully clamped orthotropic rectangular thin plates. Appl Math Lett 22(12):1821-1827

3. Zhong Y, Li R (2009) Exact bending analysis of fully clamped rectangular thin plates subjected to arbitrary loads by new symplectic approach. Mech Res Commun 36(6):707-714

4. Mbakogu FC, Pavlovic MN (2000) Bending of clamped orthotropic rectangular plates: a variational symbolic solution. Comput Struct 77(2): 117-128

5. Wolfram S (2003) The Mathematica Book, 5th edn. Wolfram Media/Cambridge University Press, Champaign

6. Dalaei M, Kerr AD (1995) Analysis of clamped rectangular orthotropic plates subjected to a uniform lateral load. Int J Mech Sci 37(5):527-535 
7. Aghdam MM, Falahatgar SR (2003) Bending analysis of thick laminated plates using extended kantorovich method. Compos Struct 62(3-4):279-283

8. Donning BM, Liu WK (1998) Meshless methods for sheardeformable beams and plates. Comput Method Appl Mech Eng 152(1-2):47-71

9. Papargyri-Beskou S, Giannakopoulos AE, Beskos DE (2010) Variational analysis of gradient elastic flexural plates under static loading. Int J Solids Struct 47(20):2755-2766

10. Shen PC, He PX (1995) Bending analysis of rectangular moderately thick plates using spline finite-element method. Comput Struct 54(6): 1023-1029

11. Huang JY (2004) Uniformly valid asymptotic solutions of the nonlinear unsymmetrical bending for orthotropic rectangular thin plate of four clamped edges with variable thickness. Appl Math Mech Engl 25(7):817-826

12. Civalek O (2007) Three-dimensional vibration, buckling and bending analyses of thick rectangular plates based on discrete singular convolution method. Int J Mech Sci 49(6):752-765

13. Cotta RM (1993) Integral transforms in computational heat and fluid flow. CRC Press, Boca Raton

14. Cotta RM, Mikhailov MD (1997) Heat conduction-lumped analysis, integral transforms, symbolic computation. Wiley, Chichester

15. Cotta RM (1998) The integral transform method in thermal and fluids science and engineering. Begell House, New York

16. Ma JK, Su J, Lu CH, Li JM (2006) Integral transform solution of the transverse vibration of an axial moving string. J Vib Meas Diagn 26(117):104-107

17. An C, Su J (2011) Dynamic response of clamped axially moving beams: Integral transform solution. Appl Math Comput 218(2):249-259

18. Matt CFT (2013) Combined classical and generalized integral transform approaches for the analysis of the dynamic behavior of a damaged structure. Appl Math Model 37(18-19):8431-8450
19. Matt CFT (2013) Simulation of the transverse vibrations of a cantilever beam with an eccentric tip mass in the axial direction using integral transforms. Appl Math Model 37(22):9338-9354

20. An C, Su J (2015) Dynamic response of axially moving Timoshenko beams: integral transform solution. Appl Math Mech Engl 35(11):1421-1436

21. An C, Su J (2014) Dynamic analysis of axially moving orthotropic plates: Integral transform solution. Appl Math Comput 228:489-507

22. Gu JJ, An C, Duan ML, Levi C, Su J (2013) Integral transform solutions of dynamic response of a clamped-clamped pipe conveying fluid. Nucl Eng Des 254:237-245

23. Gu JJ, An C, Levi C, Su J (2012) Prediction of vortex-induced vibration of long flexible cylinders modeled by a coupled nonlinear oscillator: Integral transform solution. J Hydrodyn 24(6):888-898

24. Matt CFT (2009) On the application of generalized integral transform technique to wind-induced vibrations on overhead conductors. Int J Numer Methods Eng 78(8):901-930

25. Timoshenko SP, Woinowsky-Krieger SW (1959) Theory of Plates and Shells. McGraw-Hill, New York

26. Wojtaszak IA (1937) The calculation of maximum deflection, moment, and shear for uniformly loaded rectangular plates with clamped edges. J Appl Mech 4:173-176

27. ABAQUS (2009) User's and Theory Manuals Version 6.9-1, Hibbit, Karlsson and Sorensen Inc, RI, USA

28. Szilard R (1974) Theory and analysis of plates: classical and numerical methods. Prentice-Hall, New Jersey

29. Guerrero JSP, Cotta RM (1996) Benchmark integral transform results for flow over a backward-facing step. Comput Fluids 25(5):527-540

30. IMSL (2003) IMSL Fortran Library version 5.0, MATH/ LIBRARY, vol 2, Visual Numerics Inc, Houston, TX 